\title{
The kinematics of the white dwarf population from the SDSS DR12
} \author{
K. Freeman and T. Zwitter \\ Department of Astronomy, University of Virginia, \\ Charlottesville, VA 22904-4325, USA \\ email: ba7t@virginia.edu
}

B. Anguiano, A. Rebassa-Mansergas, E. García-Berro, S. Torres,

\begin{abstract}
We use the Sloan Digital Sky Survey Data Release 12, which is the largest available white dwarf catalog to date, to study the evolution of the kinematical properties of the population of white dwarfs in the Galactic disc. We derive masses, ages, photometric distances and radial velocities for all white dwarfs with hydrogen-rich atmospheres. For those stars for which proper motions from the USNO-B1 catalog are available the true three-dimensional components of the stellar space velocity are obtained. This subset of the original sample comprises 20,247 objects, making it the largest sample of white dwarfs with measured three-dimensional velocities. Furthermore, the volume probed by our sample is large, allowing us to obtain relevant kinematical information. In particular, our sample extends from a Galactocentric radial distance $R_{\mathrm{G}}=7.8 \mathrm{kpc}$ to $9.3 \mathrm{kpc}$, and vertical distances from the Galactic plane ranging from $Z=-0.5 \mathrm{kpc}$ to $0.5 \mathrm{kpc}$. We examine the mean components of the stellar threedimensional velocities, as well as their dispersions with respect to the Galactocentric and vertical distances. We confirm the existence of a mean Galactocentric radial velocity gradient, $\partial\left\langle V_{\mathrm{R}}\right\rangle / \partial R_{\mathrm{G}}=-3 \pm 5 \mathrm{~km} \mathrm{~s}^{-1} \mathrm{kpc}^{-1}$. We also confirm North-South differences in $\left\langle V_{\mathrm{z}}\right\rangle$. Specifically, we find that white dwarfs with $Z>0$ (in the North Galactic hemisphere) have $\left\langle V_{\mathrm{z}}\right\rangle<0$, while the reverse is true for white dwarfs with $Z<0$. The age-velocity dispersion relation derived from the present sample indicates that the Galactic population of white dwarfs may have experienced an additional source of heating, which adds to the secular evolution of the Galactic disc.
\end{abstract}

Keywords. (stars:) white dwarfs; Galaxy: general; Galaxy: evolution; Galaxy: kinematics and dynamics; (Galaxy:) solar neighborhood; Galaxy: stellar content

\section{Introduction}

The ensemble properties of the white dwarf population are recorded in the white dwarf luminosity function, which therefore carries crucial information about the star formation history, the initial mass function, or the nature and history of the different components of our Galaxy — see the recent review of García-Berro \& Oswalt (2016) for an extensive list of possible applications, as well as for updated references on this topic. Among these applications perhaps the most well known of them is that white dwarfs are frequently used as reliable cosmochronometers. The need of a complete sample with accurate measurements of true space velocities, distances, masses and ages, is crucial for studying the evolution of our Galaxy. In this sense, it is worth emphasizing that little progress has been done to use the Galactic white dwarf population to unravel the evolution of the Galactic disc studying the age-velocity relationship (AVR). Since white dwarfs are excellent natural clocks we use them to compute accurate ages and in this way we determine the AVR in the solar vicinity. All this allows us to investigate the kinematic evolution of the Galactic disc (see Anguiano et al. (2017) for details). 

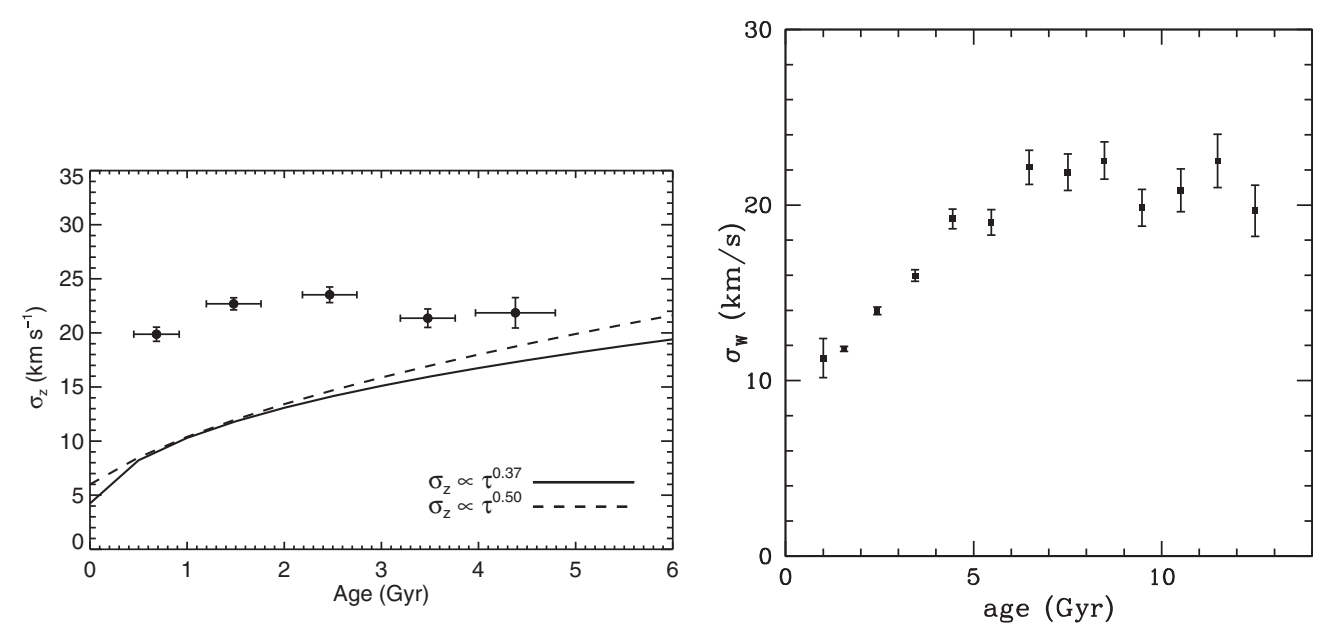

Figure 1. Left: Age-velocity dispersion relation for the vertical component of the velocity using the ages derived using the IFMR of Catalán et al. (2008). Dynamical heating functions following a power law, $\sigma_{\mathrm{z}} \propto \tau^{\alpha}$, with $\alpha=0.37$ (solid line) and 0.50 (dashed line) are also represented (Anguiano et al. (2017)). Right: Vertical velocity dispersion $\sigma_{W}$ vs stellar age for the solar neighborhood, for stars with $[\mathrm{Fe} / \mathrm{H}]>-0.3$; data from Casagrande et al. (2011).

\section{Age-velocity dispersion relation}

The age-velocity dispersion relation derived from the present WD sample indicates that the Galactic population of white dwarfs may have experienced an additional source of heating, which adds to the secular evolution of the Galactic disc (Fig. 1). The GCS stars in the age range of our WDs $(<4$ Gyr) have velocity dispersions that are clearly less than the $23 \mathrm{~km} \mathrm{~s}^{-1}$ found for the colder component of the WDs. The origin of this heating mechanism remains unclear. One possibility is that some thick disc-halo stars may have contaminated the younger bins of the AVR, thus making the age-velocity dispersion hotter. Another possibility is that there is an intrinsic dispersion for these stars. Given that white dwarfs are the final products of the evolution of stars with low and intermediate masses they might have experienced a velocity kick of $\sim 10 \mathrm{~km} \mathrm{~s}^{-1}$ during the final phases of their evolution Davis et al. (2008). It is also important to keep in mind that, although we have excluded in our analysis all white dwarfs with masses smaller than $0.45 \mathrm{M}_{\odot}$, because they are expected to be members of close binaries, a possible contamination by close pairs — of the order of 10 per cent Badenes \& Maoz (2012), Maoz \& Hallakoun (2017) - may influence the age-velocity dispersion relation. Finally, our sample of DA white dwarfs is not homogeneously distributed on the sky, but instead is drawn from the SDSS. This means that, possibly, important selection effects could affect the results. All these alternatives need to be carefully explored employing detailed population synthesis models. We postpone this study for a future publication.

\section{References}

Anguiano, B., Rebassa-Mansergas, A., Garcia-Berro, E., et al. 2017, arXiv:1703.09152

Badenes, C. \& Maoz, D. 2012, ApJ, 749, L11

Casagrande, L., Schönrich, R., Asplund, M., et al. 2011, A\&SA, 530, A138

Catalán, S., Isern, J., García-Berro, E., \& Ribas, I. 2008, MNRAS, 387, 1693

Davis, D. S., Richer, H. B., King, I. R., et al. 2008, MNRAS, 383, L20

García-Berro, E. \& Oswalt, T. D. 2016, NewAR, 72, 1

Maoz, D. \& Hallakoun, N. 2017, MNRAS, 467, 1414 